TRANSACTIONS OF THE

AMERICAN MATHEMATICAL SOCIETY

Volume 313, Number 1, May 1989

\title{
LOCAL PROPERTIES OF SECANT VARIETIES IN SYMMETRIC PRODUCTS. PART I
}

\author{
MARK E. HUIBREGTSE AND TRYGVE JOHNSEN
}

\begin{abstract}
Let $L$ be a line bundle on an abstract nonsingular curve $C$, let $V \subset H^{0}(C, L)$ be a linear system, and denote by $C^{(d)}$ the symmetric product of $d$ copies of $C$. There exists a canonically defined $C^{(d)}$-bundle map:

$$
\sigma: V \otimes \mathscr{O}_{C^{(d)}} \rightarrow E_{L},
$$

where $E_{L}$ is a bundle of rank $d$ obtained from $L$ by a so-called symmetrization process. The various degenerary loci of $\sigma$ can be considered as subsecant schemes of $C^{(d)}$. Our main result, Theorem 4.2, is given in $\S 4$, where we obtain a local matrix description of $\sigma$ valid (also) at points on the diagonal in $C^{(d)}$, and thereby we can determine the completions of the local rings of the secant schemes at arbitrary points. In $\S 5$ we handle the special case of giving a local scheme structure to the zero set of $\sigma$.
\end{abstract}

\section{INTRODUCTION}

For a curve $C \subset \mathbf{P}^{n}$ one is often interested in studying the linear subspaces of $\mathbf{P}^{n}$ containing divisors on $C$ of a certain degree. It is then convenient to find a variety (called a secant variety) which parametrizes the situations where an exceptional secancy by a linear subspace of some fixed dimension occurs. A typical example is to describe the trisecant lines for a curve in $\mathbf{P}^{3}$; another example may be to describe the 4-secant planes for a curve in $\mathbf{P}^{4}$.

When dealing with these problems there are at least two main strategies at hand: One can work in the Grassmannian parametrizing linear subspaces of $\mathbf{P}^{n}$ of some fixed dimension. This was done in [G-P] in the case $C \subset \mathbf{P}^{3}$. One can also work in $C^{(d)}$, the $d$ th symmetric product of $C$, parametrizing effective divisors on $C$ of the fixed degree $d$. For an example of this, see [A-C-G-H, Chapter VIII]. In addition there are other methods and setups, like the one in [L], which was applied in [WL] to obtain local results. See also [LB].

In our paper we will use the second approach and work with the symmetric product $C^{(d)}$. Our goal is to give local results about secant varieties, which will be subvarieties of $C^{(d)}$, and we will not take up any global questions. Roughly speaking we will show how the local geometry of $C$ at points of secancy determines the local geometry of our secant varieties at the divisors in question.

Received by the editors September 10, 1987.

1980 Mathematics Subject Classification (1985 Revision). Primary 14M15, 14H45, 14B12.

Key words and phrases. Secant varieties of curves, local geometry. 
A lot of problems like this are completely trivial at points off the weak diagonal in $C^{(d)}$, that is, at points representing divisors without repeated points. We will show how such problems can be solved at points on the weak diagonal in $C^{(d)}$.

Like other authors working with secant varieties on $C^{(d)}$, we will define these varieties as degeneration loci (schemes) of a certain $C^{(d)}$-bundle map:

$$
\sigma: V \otimes \mathscr{O}_{C^{(d)}} \rightarrow E_{L}
$$

Here $V \subset H^{0}(C, L)$ is the linear system on $C$ defining the map $C \rightarrow \mathbf{P}^{n}$, and $E_{L}$ is a $C^{(d)}$-bundle of rank $d$ on $C^{(d)}$ obtained from the line bundle $L$ on $C$ by a so-called symmetrization process. $C$ is regarded as an abstract nonsingular curve.

Part I of this paper is mainly devoted to giving a local matrix description of the map $\sigma$ at points on as well as off the weak diagonal in $C^{(d)}$, for arbitrary $d$. This matrix description will give us enough information to describe power series that determine the completions of the local rings of our secant varieties at the points in question. We will not need the assumption that $V$ is very ample or even base point free in order to describe the map $\sigma$ this way, and we need no assumptions on the characteristic of our ground field. The geometric problems mentioned in the beginning can thus be seen as a motivation for, rather than the essence of, our work, which is purely algebraic. Our treatment is based on the approach and the methods in [Ma-Ma and $\mathrm{Ma}$ ]

In $\S 2$ we list some standard facts about the symmetrization map:

$$
\sigma: V \otimes \mathscr{O}_{C(d)} \rightarrow E_{L}
$$

In $\S 3$ we treat the special case $L=\Omega$, the canonical sheaf of differentials on $C$. We show how a local matrix representation of $\sigma$ can be obtained in this special case. The key point here is the differential analogue of the Newton identities; see [Ma-Ma, p. 225].

In $\S 4$ we show how the results for $L=\Omega$ can be generalized to be valid for any line bundle on $C$. We state our main result, Theorem 4.2 , which gives the desired matrix description of the map $\sigma$ and determines the formal completion of the local rings of our secant varieties.

In $\S 5$ we apply Theorem 4.2 to determine local multiplicities of the zero scheme of $\sigma$.

At last we use our steup from $\S 4$ to reproduce two well-known formulas:

In $\S 6$ we calculate the contribution of a cusp singularity to the total number $\frac{1}{2}(d-1)(d-2)-g$ of singularities of a plane curve of degree $d$ and genus $g$. In $\S 7$ we give the multiplicity of a Weierstrass point of an arbitrary linear system.

Part II is devoted to applications of Theorem 4.2 to various geometric problems. For more details, see the introduction to that part. 
Acknowledgment. Most of the work on this paper was done while the authors were guests at Massachusetts Institute of Technology. We would like to say thank you for a pleasant stay.

\section{SYMMETRIZATION OF LINE BUNDLES}

Let $C$ be an abstract nonsingular curve over some field $K$ of arbitrary characteristic. Denote by $C^{d}$ the $d$-fold Cartesian product of $C$ and by $C^{(d)}$ the $d$-fold symmetric product.

Let $V \subset H^{0}(C, L)$ be a linear system on $C$, where $L$ is some line bundle. We will define and study the symmetrized bundle $E_{L}$ and the canonical $C^{(d)}$. bundle map

$$
\sigma: V \otimes_{K} \mathscr{O}_{C^{(d)}} \rightarrow E_{L}
$$

which was mentioned in the introduction. In particular we will find a local analytic matrix description of $\sigma$ at an arbitrary effective divisor $D$ in $C^{(d)}$.

Set $D=\sum_{i=1}^{k} d_{i} P_{i}$, where $\sum_{i=1}^{k} d_{i}=d$ and the $P_{i}$ are distinct points on $C$. Our matrix description will depend only on local analytic parametrizations at $P_{1}, \ldots, P_{k}$ of some chosen set of sections spanning $V$.

$A$ description of $\sigma: V \otimes C^{(d)} \rightarrow E_{L}$. (See also [A-C-G-H, p. 340].) Consider the diagrams

$$
C^{(d)} \stackrel{p}{\longleftarrow} F \subset C^{(d)} \times C \stackrel{q}{\longrightarrow} C
$$

where $F$ is the universal divisor $\{(D, P) \mid D$ contains $P\}$ and

$$
C^{(d)} \stackrel{\chi}{\longleftarrow} C^{d} \stackrel{\pi_{i}}{\longrightarrow} C
$$

where $\pi_{i}$ is the $i$ th projection from $C^{d}$ to $C$, and $\chi$ is the natural map onto $C^{(d)}$.

Set $L[d]=\bigoplus_{i=1}^{d} \pi_{i}^{*} L$. Clearly $L[d]$ is a locally free sheaf on $C^{d}$ of rank $d$. Let $\varepsilon_{L}$ be the sheaf on $C^{(d)}$ whose sections on an open set $U$ are the $G$-invariant sections of $L[d]$ on $\chi^{-1}(U)$, where $G$ is the Galois covering-map group of $\chi$.

Set $E_{L}=p_{*} q^{*} L$. It can be shown that $E_{L}=\varepsilon_{L}$, and it is a locally free sheaf of rank $d$. This is essentially Proposition $1, \mathrm{p} .781$, in [Ma]. In particular each section $S$ of $L$ gives rise to a section $S[d]=\sum_{i=1}^{d} \pi_{i}^{*} S$ of $L[d]$ on $C^{d}$. The section $S[d]$ is $G$-invariant since $G$ acts by rearranging the summands of $S[d]$. Hence $S$ gives rise to a section $S_{d}$ of $\varepsilon_{L}=E_{L}$ on $C^{(d)}$.

Definition. The map

$$
\sigma: V \otimes_{K} \mathscr{O}_{C^{(d)}} \rightarrow E_{L}
$$

is defined by letting $S$ be mapped to $S_{d}$ for each section $S$ in $V$.

Remark. When $D$ consists of $d$ distinct points, $\sigma$ "simply evaluates" $S$ at these points. 
A local simplification. When $D=\sum_{i=1}^{k} d_{i} P_{i}$, where the $P_{i}$ are distinct, there are $d ! / \pi_{i=1}^{k} d_{i}$ ! points in $C^{d}$ that are mapped to $D$ by the canonical map $\chi$. Let $D^{\prime \prime}$ in $C^{d}$ be $\left(P_{1}, \ldots, P_{1}, \ldots, P_{k}, \ldots, P_{k}\right)$, where the point $P_{i}$ is taken $d_{i}$ times, for $i=1, \ldots, k$. Clearly $D^{\prime \prime}$ is in the fiber of $\chi$ over $D$. Let $\chi_{d_{1}, \ldots, d_{k}}$ be the partial symmetrization map which maps $\left(Q_{1}, \ldots, Q_{d}\right) \in C^{d}$ to $\left(\sum_{i=1}^{d_{1}} Q_{i}, \ldots, \sum_{i=d_{k-1}+1}^{d} Q_{i}\right) \in C^{\left(d_{1}\right)} \times \cdots \times C^{\left(d_{k}\right)}$.

Definition. The sheaf $L_{d_{1}, \ldots, d_{k}}$ on $C^{\left(d_{1}\right)} \times \cdots \times C^{\left(d_{k}\right)}$ is the sheaf whose sections on an open set $U$ are the invariant sections of $L[d]$ on $\chi_{d_{1}, \ldots, d_{k}}^{-1}(U)$ under the Galois group associated to $\chi_{d_{1}, \ldots, d_{k}}$.

We see that each (global) section $S$ of $L$ gives rise to a section $S_{d_{1}, \ldots, d_{k}}$ of $L_{d_{1}, \ldots, d_{k}}$.

Definition. The map

$$
\sigma^{\prime}: V \otimes \mathscr{O}_{C^{\left(d_{1}\right)} \times \cdots \times C^{\left(d_{k}\right)}} \rightarrow L_{d_{1}, \ldots, d_{k}}
$$

is defined by setting

$$
\sigma^{\prime}(S)=S_{d_{1}, \ldots, d_{k}} \text { for each section } S \text { in } V .
$$

We now make the following observation:

Observation 2.1. Study the following commutative diagram:

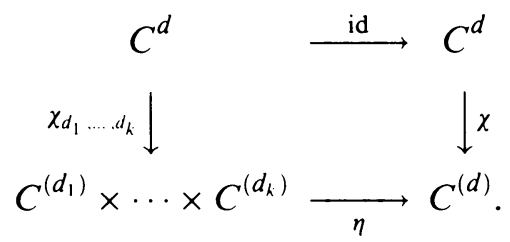

Denote by $D^{\prime}$ the point $\left(d_{1} P_{1}, \ldots, d_{k} P_{k}\right)$ of $C^{\left(d_{1}\right)} \times \cdots \times C^{\left(d_{k}\right)}$. The natural map $\eta$ is a local analytic isomorphism at $D^{\prime}, D$. Moreover, $\eta$ induces a local analytic isomorphism of the bundle maps $\sigma^{\prime}$ and $\sigma$ at $D^{\prime}$ and $D$, respectively. Explanation. The first statement is a standard fact. See [Ma-Ma, p. 226]. Since $\sigma^{\prime}$ and $\sigma$ are derived from the natural $C^{d}$-bundle map

$$
V \otimes_{K} \mathscr{O}_{C^{d}} \rightarrow L[d]
$$

via $\chi_{d_{1}, \ldots, d_{k}}$ and $\chi$, respectively, the last statement also holds.

In $\S 3$ we will use Observation 2.1 to obtain a local matrix representation of $\sigma$ in the case where $L$ is the canonical sheaf on $C$.

\section{THE SYMMETRIZATION OF DIFFERENTIALS}

We will first consider the process of symmetrization of differentials; hence we will assume that $V \subset H^{0}(C, \Omega)$, where $\Omega$ is the sheaf of differentials on 
$C$. In $\S 4$ we will explain how this special case gives us enough information to describe the map $\sigma$ locally for a general line bundle $L$ on $C$.

Let $\varphi$ be a holomorphic differential on $C$, and set $D=\sum_{i=1}^{k} d_{i} P_{i}$ as usual. To $\varphi$ there corresponds a section $S^{\varphi}$ of $\Omega$, which gives rise to a section $S^{\varphi}[d]$ of $\Omega[d]$ on $C^{d}$. The section $S^{\varphi}[d]$ can be interpreted as a 1 -form $\varphi[d]$ on $C^{d}$, which is invariant with respect to any subgroup of the permutation group acting on $C^{d}$. Let $t_{i}$ be a local parameter of $C$ at $P_{i}$, and let $\varphi$ be given locally at $P_{i}$ as

$$
\left(\sum_{j \geq 0} a_{i, j} t_{i}^{j}\right) d t_{i}, \quad i=1, \ldots, k .
$$

Then the 1 -form $\varphi[d]$ is given locally at $D^{\prime \prime}=\left(P_{1}, \ldots, P_{1}, \ldots, P_{k}, \ldots, P_{k}\right)$ as

$$
\sum_{i=1}^{k} \sum_{j \geq 0} a_{i, j}\left(\sum_{l=1}^{d_{i}} t_{i, l}^{j} d t_{i, l}\right) .
$$

In (3.1) the symbol $t_{i, l}$ is short for $\pi_{d_{1}+\cdots+d_{i-1}+l}^{*}\left(t_{i}\right)$; see diagram (2.2). The $t_{i, l}$ can be viewed as formal replicas of $t_{i}$, for $l=1, \ldots, d_{i}, i=1, \ldots, k$.

A partially symmetrized section $S_{d_{1}, \ldots, d_{k}}^{\varphi}$ of $\Omega_{d_{1}, \ldots, d_{k}}$ corresponds to a partially symmetrized 1 -form $\varphi_{d_{1}, \ldots, d_{k}}$ on $C^{\left(d_{1}\right)} \times \cdots \times C^{\left(d_{k}\right)}$. A set of regular parameters for $C^{\left(d_{1}\right)} \times \cdots \times C^{\left(d_{k}\right)}$ at $D^{\prime}=\left(d_{1} P_{1}, \ldots, d_{k} P_{k}\right)$ is

$$
\bigcup_{i=1}^{k}\left\{s_{i, 1}, \ldots, s_{i, d_{i}}\right\}
$$

where $s_{i, j}$ is the $j$ th elementary symmetric function in the formal variables $t_{i, 1}, \ldots, t_{i, d_{i}}$. See [Ma-Ma, p. 226].

The sums $\left(\sum_{l=1}^{d_{i}} t_{i, l}^{j} d t_{i, l}\right)$ appearing in (3.1) are symmetric in $\left\{t_{i, 1}, \ldots\right.$, $\left.t_{i, d_{i}}\right\}$. Hence (3.1) can be expressed in terms of $\left\{s_{i, 1}, \ldots, s_{i, d_{i}}\right\}$ and the differential symbols $\left\{d s_{i, 1}, \ldots, d s_{i, d_{i}}\right\}$ for $i=1, \ldots, k$. Clearly this will be the expression of $\varphi_{d_{1}, \ldots, d_{k}}$ locally at $D^{\prime}$. In the next paragraph we will study these partially symmetrized 1 -forms more closely.

Partially symmetrized differentials in terms of the elementary symmetric functions. We will now express (3.1) in terms of $\bigcup_{i=1}^{k}\left\{s_{i, 1}, \ldots, s_{i, d_{t}}\right\}$, and we will treat each of the $k$ "main summands" in (3.1) separately. Hence we may assume $D=d P$, and we have one set $\left\{s_{1}, \ldots, s_{d}\right\}$ of elementary symmetric functions in $\left\{t_{i}, \ldots, t_{d}\right\}$. The $t_{i}$ are formal replicas of the local parameter $t$ of $C$ at $P$. In this case (3.1) reduces to

$$
\sum_{j \geq 0} a_{j} \sum_{l=1}^{d} t_{l}^{j} d t_{l}
$$


Set $T_{j}^{\prime}=\sum_{l=1}^{d} t_{l}^{j} d t_{l}$, and let $T_{j}$ be the corresponding 1-form on $C^{(d)}$, which means that $T_{j}$ is $T_{j}^{\prime}$ expressed in terms of the $s_{i}$. We have the following key formula, given for example in [Ma-Ma p. 225]:

$$
s_{k} T_{0}-s_{k-1} T_{1}+\cdots+(-1)^{k} s_{0} T_{k}=d s_{k+1} .
$$

Formula (3.2) is valid in all characteristics and for all integers $k$ with the convention tht $s_{0}=1, s_{i}=T_{i}=0$ when $i<0, s_{i}=0$ when $i>d$. Using Formula (3.2) recursively one gets

$$
\begin{aligned}
& T_{0}=d s_{1}, \\
& T_{1}=s_{1} d s_{1}-d s_{2}, \\
& T_{2}=\left(s_{1}^{2}-s_{2}\right) d s_{1}-s_{1} d s_{2}+d s_{3} \text { and so on. }
\end{aligned}
$$

Let the symbol $(\underline{s})$ be short for $\left(s_{1}, \ldots, s_{d}\right)$. In general one has

Proposition 3.1.

$$
T_{j}=W_{j}(\underline{s}) d s_{1}-W_{j-1}(\underline{s}) d s_{2}+\cdots+(-1)^{d-1} W_{j-d+1}(\underline{s}) d s_{d}
$$

where

$$
W_{j}(\underline{s})=\operatorname{det}\left[\begin{array}{cccccc}
s_{1} & s_{2} & \ldots & & & s_{j} \\
1 & s_{1} & \ldots & & & s_{j-1} \\
0 & 1 & \ldots & & & \\
\vdots & & \ddots & & & \vdots \\
0 & & \ldots & 0 & 1 & s_{1}
\end{array}\right]
$$

when $j \geq 1$ and $W_{0}(\underline{s})=1, W_{j}(\underline{s})=0, j<0$.

Proof. Set $T_{j}=\sum_{i=1}^{d} W_{i, j}(\underline{s}) d s_{i}$, where $W_{i, j}(\underline{s}) \in k[(\underline{s})]$. Since the $s_{i}$, for $i=1, \ldots, d$, are local parameters at $D$, the $W_{i, j}(\underline{s})$ are uniquely determined, and we see that for fixed $i$ the $W_{i, k}(\underline{s})$ satisfy the following recursion formula, which is derived from formula (3.2):

$$
W_{i, k}(\underline{s})=(-1)^{k-1}\left(s_{k} W_{i, 0}(\underline{s})-s_{k-1} W_{i, 1}(\underline{s})+\cdots+(-1)^{k-1} s_{1} W_{i, k-1}(\underline{s})\right) .
$$

We define $W_{j}(\underline{s})=W_{1, j}(\underline{s})$.

Observe that for $i=1$ the recursion formula (3.3) "matches" expanding the determinant in Proposition 3.1 along the last column. Hence the $W_{1, j}(\underline{s})$ are as stated in Proposition 3.1.

From formula (3.2) we see that

$$
W_{i, i-1}(\underline{s})=(-1)^{i-1} \text { and } W_{i, j}(\underline{s})=0 \text { for } j \leq i-2 .
$$

This implies that

$$
W_{i+1, j+1}(\underline{s})=-W_{i, j}(\underline{s})
$$

for $1 \leq i \leq d-1$ and arbitrary $j$, since the recursion using formula (3.3) starts one step later for $i+1$ than for $i$ and with opposite sign.

The last identity gives that the $W_{i, j}(\underline{s})$ for arbitrary $i$ are as stated in Proposition 3.1 , since we know that the $W_{1, j}$ are. This completes our proof. 
Remark. We have

$$
W_{k}(\underline{s})=\sum s_{1}^{i_{1}} \cdots s_{d}^{i_{d}} \cdot(-1)^{\sum_{j=1}^{d} i_{j}(j-1)} \cdot \frac{\left(i_{1}+\cdots+i_{d}\right) !}{i_{1} ! \cdots \cdot i_{d} !}
$$

where the first sum is taken over those $\left(i_{1}, \ldots, i_{d}\right)$ such that $\sum_{j=1}^{d} j i_{j}=k$.

We also have

$$
W_{j}\left(s_{1}\left(t_{1}, \ldots, t_{d}\right), \ldots, s_{d}\left(t_{1}, \ldots, t_{d}\right)\right)
$$

is the sum of all (monic) monomials of degree $j$ in $\left\{t_{1}, \ldots, t_{d}\right\}$.

Furthermore we have

$$
W_{j}(\underline{s})=\Delta_{1, \ldots, 1}(\underline{s})
$$

in the sense of [F, p. 264].

Formulas (3.4), (3.5) can be proved, for example, by using formula (3.3).

Corollary 3.2. If $\varphi$ is a holomorphic differential on $C$ given locally at $P$ as $\left(\sum_{j \geq 0} a_{j} t^{j}\right) d t$, then the symmetrized 1 -form $\varphi_{d}$ on $C^{(d)}$ is given locally at $D=d P$ as

$$
\sum_{j \geq 0} a_{j} T_{j}=\sum_{j \geq 0} a_{j}\left[W_{j}(\underline{s}) d s_{1}-W_{j-1}(\underline{s}) d s_{2}+\cdots+(-1)^{d-1} W_{j-d+1}(\underline{s}) d s_{d}\right] .
$$

If $D=\sum_{i=1}^{k} d_{i} P_{i}$, where $\sum_{i=1}^{d} d_{i}=d$, then $\varphi_{d_{1}, \ldots, d_{k}}$ is given locally at $D^{\prime}=$ $\left(d_{1} P_{1}, \ldots, d_{k} P_{k}\right)$ as the sum of $k$ sums of the form (3.6). Choose the $d s_{i}$, for $i=1, \ldots, d$, as a basis of the fiber of $E_{\Omega}$ over $d P$. A local parametrization of the section $S_{d}^{\varphi}$ of $E_{\Omega}$ on $C^{(d)}$ at $d P$ in terms of coordinates $Y_{1}, \ldots, Y_{d}$ relative to this basis is

$$
\left\{Y_{1}=\sum_{j \geq 0} a_{j} W_{j}(\underline{s}), \ldots, Y_{d}=\sum_{j \geq 0} a_{j} W_{j-d+1}(\underline{s})\right\} .
$$

Proof. Everything but the last assertion is just a restatement of Proposition 3.1 using the linearity of the (partial) symmetrization map.

The last assertion holds since $\left\{s_{1}, \ldots, s_{d}\right\}$ is a set of regular parameters of $C^{(d)}$ at $d P$. Hence the 1 -forms $d s_{1}, \ldots, d s_{d}$ form a local basis for the 1-forms on $C^{(d)}$ at $d P$.

If $S$ is a basis element of $V$, we see from Corollary 3.2 that the entries

$$
\sum_{j \geq 0} a_{j} W_{j}(\underline{s}), \ldots, \sum_{j \geq 0} a_{j} W_{j-d+1}
$$

can be taken to form one of the columns of a local matrix description of $C^{(d)}$ bundle map

$$
\sigma: V \otimes \mathscr{O}_{C^{(d)}} \rightarrow E_{\Omega}
$$

at $d P$. The generalization to the case $D=\sum_{i=1}^{k} d_{i} P_{i}$ is obvious when working with partially symmetrized 1 -forms on $C^{\left(d_{1}\right)} \times \cdots \times C^{\left(d_{k}\right)}$ and using Observation 2.1. 


\section{A LOCAL DESCRIPTION OF SECANT VARIETIES}

In $\S 2$ we defined the canonical bundle map

$$
\sigma: V \otimes \mathscr{O}_{C^{(d)}} \rightarrow E_{L}
$$

In $\S 3$ we assumed $L=\Omega$, and we described how local parametrizations of the sections of $V$ at $P_{1}, \ldots, P_{k}$ gives rise to a matrix description of $\sigma$ in terms of local analytic coordinates of $C^{\left(d_{1}\right)} \times \cdots \times C^{\left(d_{k}\right)}$ at $D^{\prime}=\left(d_{1} P_{1}, \ldots, d_{k} P_{k}\right)$.

It is clear that this matrix description depends only on the parametrizations of the sections of $V$ and, once the parametrizations are given, is independent of whether the sections of $V$ correspond to differentials or not, that is, whether $L=\Omega$ or not.

This was explained in [Ma, p. 783], the proof of Proposition 2 of that paper. Hence our local matrix description of $\sigma$ is valid for any linear system $V$, where the local parametrizations of the sections (divisors) of $V$ are equal to those of some globally holomophic differentials on $C$.

The last condition does not necessarily hold for an arbitrary linear system $V$. However, we give the following remark. Set $D=\sum_{i=1}^{k} d_{i} P_{i}$.

Remark 4.1. (a) In the symmetrization process described in $\S 3$ we treated each point $P_{i}$ separately.

(b) The symmetrization process can be carried out even if the differentials we treat are not globally holomorphic. For each point $P_{i}$ contained in $D$ we can describe an analogous symmetrization process for any differential which is holomorphic on a neighborhood $U_{i}$ of $P_{i}$. We can produce a symmetrized 1 -form on $U_{i}^{\left(d_{i}\right)}$ which is a neighborhood of $d_{i} P_{i}$ on $C^{\left(d_{i}\right)}$.

Remark 4.1 and the comment in [Ma, p. 783] imply together that for a section $S$ of an arbitrary line bundle $L$, where $S$ is given locally at $P_{i}$ as $\sum_{j \geq 0} a_{i, j} t_{i}^{j}$, we can describe the map

$$
V \otimes \mathscr{O}_{C^{\left(d_{i}\right)}} \rightarrow E_{L}
$$

locally simply by pretending that we are symmetrizing a differential given locally at $P_{i}$ as

$$
\left(\sum_{j \geq 0} a_{i, j} t_{i}^{j}\right) d t_{i} .
$$

Hence it is clear that our local matrix description of $\sigma$ in $\S 3$ holds for $V \subseteq$ $H^{0}(C, L)$ for any line bundle $L$ on $C$.

We then obtain

Theorem 4.2. Let $X_{0}, \ldots, X_{n}$ be sections spanning the linear system $V \subset$ $H^{0}(C, L)$ for a line bundle $L$. Choose $t_{i}$ as a local parameter for $C$ at $P_{i}$, for $i=1, \ldots, k$, and let

$$
\sum_{j \geq 0} a_{r, i, j} t_{i}^{j}
$$


be local parametrizations of $X_{r}$ at $P_{i}$, for $i=1, \ldots, k$ and $r=0, \ldots, n$. Then a local matrix description of the map $\sigma: V \otimes \mathscr{O}_{C^{(d)}} \rightarrow E_{L}$ at (the point) $D=\sum_{i=1}^{k} d_{i} P_{i}$ is

$$
M=\left[\begin{array}{ccc}
\sum_{j \geq 0} a_{0,1, j} W_{j}\left(\underline{s}^{(1)}\right) & \ldots & \sum_{j \geq 0} a_{n, 1, j} W_{j}\left(\underline{s}^{(1)}\right) \\
\vdots & & \vdots \\
\sum_{j \geq 0} a_{0,1, j} W_{j-d_{1}+1}\left(\underline{s}^{(1)}\right) & \ldots & \sum_{j \geq 0} a_{n, 1, j} W_{j-d_{1}+1}\left(\underline{s}^{(1)}\right) \\
\vdots & & \vdots \\
\sum_{j \geq 0} a_{0, k, j} W_{j}\left(\underline{s}^{(k)}\right) & \ldots & \sum_{j \geq 0} a_{n, k, j} W_{j}\left(\underline{s}^{(k)}\right) \\
\vdots & & \vdots \\
\sum_{j \geq 0} a_{0, k, j} W_{j-d_{k}+1}\left(\underline{s}^{(k)}\right) & \ldots & \sum_{j \geq 0} a_{n, k, j} W_{j-d_{k}+1}\left(\underline{s}^{(k)}\right)
\end{array}\right] .
$$

The $\underline{s}^{(i)}$ indicate $d_{i}$-tuples $\left(s_{i, 1}, \ldots, s_{i, d_{i}}\right)$ of elementary symmetric functions in $d_{i}$ formal replicas of $t_{i}$. The $W_{l}$, for $l \in \mathbf{Z}$, were defined in Proposition 3.2.

Definition 4.3. For a map $f$ of $C^{(d)}$-bundles denote by $Z(f)$ the zero scheme of the map. We denote by $V_{d}^{r}$ or $D_{d-r}(\sigma)$ the zero scheme $Z\left(\Lambda^{d-r+1} \sigma\right)$, where $\sigma$ is the canonical map $V \otimes \mathscr{O}_{C^{(d)}} \rightarrow E_{L}$.

Set-theoretically we have

$$
V_{d}^{r}=\left\{D \in C^{(d)} \mid \text { rk } \sigma \leq d-r \text { at } D\right\} .
$$

Scheme-theoretically $V_{d}^{r}$ is given locally at $D$ by (the vanishing of) the $d-r+1$ minors of some matrix representation of $\sigma$ at $D$.

From Proposition 4.2 we have when $D=\sum_{i=1}^{k} d_{i} P_{i}$ :

Theorem 4.4.

$$
\hat{\mathscr{O}}_{V_{d}^{r}, D} \simeq K\left[\left[s_{1,1}, \ldots, s_{1, d_{1}}, \ldots, s_{k, 1}, \ldots, s_{k, d_{k}}\right]\right] / J,
$$

where $J$ is the ideal in $K[[(\underline{s})]]$ generated by the $d-r+1$-minors of $M$, and the $s_{i, j}$ are regarded as algebraically independent (formal) variables.

We see that $D \in V_{d}^{r}$ iff all $d-r+1$-minors of $M$ vanish modulo $\underline{m}=\left(s_{i, j}\right)$. Modulo $\underline{m}$ the matrix $M$ is

$$
B N=\left[\begin{array}{ccc}
a_{0,1,0} & \cdots & a_{n, 1,0} \\
\vdots & & \vdots \\
a_{0,1, d_{1}-1} & \cdots & a_{n, 1, d_{1}-1} \\
\vdots & & \vdots \\
a_{0, k, 0} & \cdots & a_{n, k, 0} \\
\vdots & & \vdots \\
a_{0, k, d_{k}-1} & \cdots & a_{n, k, d_{k}-1}
\end{array}\right]
$$


We will sometimes refer to $B N$ as the Brill-Noether matrix. The entries of $B N$ consist of the coefficients of $W_{0}=1$ in the infinite sums in each of the entries in $M$, since $W_{l}\left(\underline{s}^{(i)}\right)$ is contained in $\underline{m}$ when $l \geq 1$.

\section{THE SYMMETRIZED BASE POINT SCHEME OF A LINEAR SYSTEM}

In this section we will give an application of Theorem 4.2. Assume that $V$ is a linear system of rank 1 on $C$, where $V \subset H^{0}(C, L), \operatorname{deg} L=m$, and where $V$ is generated by a global section $S$. We will study the symmetrized zero (base-point) scheme $V_{d}^{d}$.

Let $\sum_{j \geq 0} a_{i, j} t_{i}^{j}$ be local parametrizations of $S$ at $P_{i}, i=1, \ldots, k$. From the Brill-Noether (column) matrix we see that $D=\sum_{i=1}^{k} d_{i} P_{i}$ is contained in $V_{d}^{d}$ iff

$$
a_{1,0}=\cdots=a_{1, d_{1}-1}=\cdots=a_{k, 0}=\cdots=a_{k, d_{k}-1}=0 .
$$

Hence $D \in V_{d}^{d}$ iff $D \leq D_{S}$, where $D_{S}$ is the element of $C^{(m)}$ corresponding to $S$. (Hence $V_{d}^{d}$ is finite.) This is well known; see, e.g., [Ma, Proposition 2, p. 783]. We will study the local lengths (multiplicities) of $V_{d}^{d}$. The total length is always $\left(\begin{array}{l}m \\ d\end{array}\right)$, roughly speaking, since $D_{S}$ contains $\left(\begin{array}{l}m \\ d\end{array}\right)$ subdivisors of degree $d$ when $D_{S}$ is off the weak diagonal in $C^{(m)}$, and since moving $S$ in $H^{0}(C, L)$ does not change total lengths. We will, however, not use any global argument like this.

We have when $D_{S}=\sum_{i=1}^{k} m_{i} P_{i}$ and $D=\sum_{i=1}^{k} d_{i} P_{i}$ :

Result 5.1. (a) The local length of $V_{d}^{d}$ at $D$ is

$$
\prod_{i=1}^{k}\left(\begin{array}{c}
m_{i} \\
d_{i}
\end{array}\right) \text {. }
$$

(Observe that

$$
\sum \prod_{i=1}^{k}\left(\begin{array}{l}
m_{i} \\
d_{i}
\end{array}\right)=\left(\begin{array}{l}
m \\
d
\end{array}\right),
$$

where the sum is taken over all divisors $D$ such that $D \leq D_{S}$. )

(b) $\hat{\mathscr{O}}_{V_{d}^{d}, D}=k[[(\underline{s})]] / I$, where

$$
I=\left(W_{m_{1}}\left(\underline{s}^{(1)}\right), \ldots, W_{m_{1}-d_{1}+1}\left(\underline{s}^{(1)}\right), \ldots, W_{m_{k}}\left(\underline{s}^{(k)}\right), \ldots, W_{m_{k}-d_{k}+1}\left(\underline{s}^{(k)}\right)\right) .
$$

The $d$ variables $s_{i, j}$ are algebraically independent, and the $W_{j}$ were defined in Proposition 3.1.

Proof. We will prove (b) and then use (b) to prove (a). Clearly we may assume $D_{S}=m P, D=d P$, and $m \geq d$ when proving (b). The ideal cutting our $V_{d}^{d}$ 
locally at $D$ is generated by the entries of $M$. The entries are

$$
\begin{array}{cccccc}
a_{m} W_{m}(\underline{s}) & + & a_{m+1} W_{m+1}(\underline{s}) & +\cdots+ & \cdots & +\cdots \\
a_{m} W_{m-1}(\underline{s}) & + & a_{m+1} W_{m}(\underline{s}) & +\cdots+ & \cdots & +\cdots \\
\vdots & & & & & \\
a_{m} W_{m-d+1}(\underline{s}) & + & a_{m+1} W_{m-d+2}(\underline{s}) & +\cdots+ & a_{m+d-1} W_{m}(\underline{s}) & +\cdots
\end{array}
$$

We see that modulo $\left(W_{m+1}(\underline{s}), W_{m+2}(\underline{s}), \ldots\right)$ these relations give a rise to the following coefficient matrix in the "variables" $W_{m-d+1}(\underline{s}), \ldots, W_{m}(\underline{s})$ :

$$
N=\left[\begin{array}{cccc}
0 & \ldots & 0 & a_{m} \\
& & a_{m} & a_{m+1} \\
\vdots & \ddots & & \vdots \\
a_{m} & a_{m+1} & \ldots & a_{m+d-1}
\end{array}\right] .
$$

By the recursion formula (3.3) we have

$$
W_{r}(\underline{s})=\sum_{i=m-d+1}^{m} P_{i}(\underline{s}) W_{i}(\underline{s})
$$

for suitable polynomials $P_{i}(\underline{s}) \in \underline{m}=\left(s_{1}, \ldots, s_{d}\right)$ when $r \geq m+1$. The summation starts at $i=m-d+1$ since in formula (3.3) we have $s_{j}=0$ when $j>d$. Hence the $d$ generators of our ideal can be expressed as linear combinations in $W_{m-d+1}(\underline{s}), \ldots, W_{m}(\underline{s})$ with coefficients in $k[[s]]$. Since the coefficient matrix thus obtained is equal to $N$ modulo $\underline{m}=\left(s_{1}, \ldots, s_{d}\right)$, we see that (b) holds since $\operatorname{det} N= \pm a_{m}^{d} \neq 0$.

We will now use (b) to prove (a). Let $I_{i}$ be the ideal $\left(W_{m_{i}}\left(\underline{s}^{(i)}\right), \ldots, W_{m_{i}-d_{i}+1}\right)$ in $k\left[\left[s_{i, 1}, \ldots, s_{i, d_{i}}\right]\right]$ for $i=1, \ldots, k$. We see that

$$
\text { colength } I=\prod_{i=1}^{k} \text { colength } I_{i} \text {, }
$$

since the $I_{i}$ are generated by functions in mutually disjoint set of variables $\left\{\underline{s}^{(i)}\right\}$. This means that we can assume again that $D_{S}=m P, D=d P$, and we must show that colength $I=\left(\begin{array}{c}m \\ d\end{array}\right)$.

We proceed by induction on $m$ and we may start with $m=d=1$. In this case $I=\left(\sum_{j \geq 1} a_{j} s_{1}^{j}\right)$, where $a_{1} \neq 0$, so colength $I=1=\left(\begin{array}{l}1 \\ 1\end{array}\right)$.

Set $W_{j}=W_{j}(\underline{s})$ for all $j$. For general $m, d$ we have

$$
\begin{aligned}
I & =\left(W_{m-d+1}, \ldots, W_{m}\right) \\
& =\left(W_{m-d+1}, \ldots, W_{m-1}, s_{1} W_{m-1}-s_{2} W_{m-2}+\cdots+(-1)^{d-1} s_{d} W_{m-d}\right) \\
& =\left(W_{m-d+1}, \ldots, W_{m-1}, s_{d} W_{m-d}\right)
\end{aligned}
$$

by formula (3.3).

Hence

$$
\begin{aligned}
\text { colength } I= & \operatorname{colength}\left(W_{m-d}, W_{m-d+1}, \ldots, W_{m-1}\right) \\
& +\operatorname{colength}\left(W_{m-d+1}, \ldots, W_{m-1}, s_{d}\right)
\end{aligned}
$$


The first summand is $\left(\begin{array}{c}m-1 \\ d\end{array}\right)$ by the induction hypothesis. The second summand is the colength of

$$
\left(W_{m-d+1}\left(s_{1}, \ldots, s_{d-1}\right), \ldots, W_{m-1}\left(s_{1}, \ldots, s_{d-1}\right)\right)
$$

in $K\left[\left[s_{1}, \ldots, s_{d-1}\right]\right]$ By the induction hypothesis this is $\left(\begin{array}{c}m-1 \\ d-1\end{array}\right)$.

Hence colength $I=\left(\begin{array}{c}m-1 \\ d\end{array}\right)+\left(\begin{array}{c}m-1 \\ d-1\end{array}\right)=\left(\begin{array}{c}m \\ d\end{array}\right)$. This proves $(\mathbf{a})$.

We will now give a generalization of Result 5.1.

Corollary 5.2. Assume that $\operatorname{rank} V=n+1$ and that $V$ is generated by sections corresponding to divisors $D_{0}, \ldots, D_{n}$ on $C$, where

$$
D_{j}=\sum_{i=1}^{k} m_{i, j} P_{i}, \quad j=0, \ldots, n .
$$

Set $D_{W}=\sum_{i=1}^{k} m_{i} P_{i}$, where $m_{i}=\min \left\{m_{i, j}\right\}_{j=0}^{n}$, for $i=1, \ldots, k$. Let $W$ be the linear system of rank 1 spanned by the section corresponding to $D_{W}$. Then

(a) $V_{d}^{d}=W_{d}^{d}$ for all $d$.

(b) The multiplicity of $V_{d}^{d}$ at $D=\sum_{i=1}^{k} d_{i} P_{i}$ is $\prod_{i=1}^{k}\left(\begin{array}{c}m_{i} \\ d_{i}\end{array}\right)$.

Proof. By Result 5.1 we see that (b) is a consequence of (a).

To prove (a) we may assume that $D_{W}=m P, D=d P$, and $D_{j}=m_{j} P, j=$ $0, \ldots, n$. By the proof of Result 5.1 the entries in the column corresponding to $D_{j}$ form the ideal $\left(W_{m_{j}}(\underline{s}), \ldots, W_{m_{j}-d+1}(\underline{s})\right)$. By formula $(3.3) W_{r}(\underline{s})$ is contained in the ideal $J=\left(W_{m}(\underline{s}), \ldots, W_{m-d+1}(\underline{s})\right)$ for all $r \geq m-d+1$. Hence

$$
\hat{\mathscr{O}}_{V_{d}^{d}, D}=\hat{\mathscr{O}}_{W_{d}^{d}, D}=K\left[\left[s_{1}, \ldots, s_{d}\right]\right] / J .
$$

Since both schemes $V_{d}^{d}$ and $W_{d}^{d}$ are zero-dimensional, this is enough to prove (a). This completes our proof.

\section{Singularities of Plane CURVES}

Here we apply Theorem 4.2 to study the well-known case of plane cusps. Assume rk $V=3$ and that $V$ is base point free. Thus $V$ defines a map

$$
\phi: C \rightarrow \bar{C} \subseteq \mathbf{P}^{2} \text {. }
$$

We can "measure" the singularities of $\bar{C}$ by studying the scheme $V_{2}^{1}$ in $C^{(2)}$. This scheme may consist of two kinds of points:

(1) Divisors $P_{1}+P_{2}$, where $P_{1} \neq P_{2}$.

(2) Divisors $2 P$.

The first ones correspond to nodes of $C$, the latter ones to cusps. If $V_{2}^{1}$ is finite, it is well known that its total length is $\frac{1}{2}(d-1)(d-2)-g$, where $d=\operatorname{deg} \bar{C}=\operatorname{deg} L$ and $g=\operatorname{genus}(C)$.

The multiplicity of $V_{2}^{1}$ at $D=2 P$. From now on we will concentrate on divisors of the type $2 P$. We will not prove anything essentially new, but we will show how our setup fits in well with traditional results. 
Denote by Mult $_{D} V_{2}^{1}$ the multiplicity or local length of $V_{2}^{1}$ at a divisor $D$. Clearly the $\delta$-invariant of $\bar{C}$ at $Q \in P^{2}$ is $\sum \operatorname{Mult}_{D} V_{2}^{1}$, where the sum is taken over those divisors $P_{1}+P_{2}$ and $2 P$ such that $\phi\left(P_{1}\right)=\phi\left(P_{2}\right)=Q$ and $\phi(P)=Q$. We will show how to find $\operatorname{Mult}_{2 P} V_{2}^{1}$, when Char $K=0$ and $K=\bar{K}$.

Choose

$$
X_{r}=\sum_{j=0}^{\infty} \alpha_{r, j} t^{j} \quad \text { for } r=0,1,2
$$

as local parametrizations at $P$ of the sections spanning $V$. We may assume $X_{0} \equiv 1$. The matrix $M$ from Theorem 4.2 is

$$
\left[\begin{array}{ccc}
1 & \sum_{j \geq 0} \alpha_{1, j} W_{j}\left(s_{1}, s_{2}\right) & \sum_{j \geq 0} \alpha_{2, j} W_{j}\left(s_{1}, s_{2}\right) \\
0 & \sum_{j \geq 1} \alpha_{1, j} W_{j-1}\left(s_{1}, s_{2}\right) & \sum_{j \geq 1} \alpha_{2, j} W_{j-1}\left(s_{1}, s_{2}\right)
\end{array}\right] .
$$

We assume $P=(1,0,0)$ and obtain

$$
\hat{\mathscr{O}}_{V_{2}^{1}, 2 P}=K\left[\left[s_{1}, s_{2}\right]\right] / I
$$

where

$$
I=\left(\sum_{j \geq 2} \alpha_{1, j} W_{j-1}\left(s_{1}, s_{2}\right), \sum_{j \geq 2} \alpha_{2, j} W_{j-1}\left(s_{1}, s_{2}\right)\right) .
$$

We have used that $\alpha_{1,1}=\alpha_{2,1}=0$ by assumption. When Char $K=0$, it is a standard fact that we may simplify our local parametrizations:

$$
X_{0}=1, \quad X_{1}=t^{n}, \quad X_{2}=\sum_{j \geq n+1} \alpha_{2, j} t^{j},
$$

where $n \geq 2$ is the multiplicity at $\phi(P)$ of the branch of $\bar{C}$ in question. The ideal $I$ reduces to

$$
\left(W_{n-1}\left(s_{1}, s_{2}\right), \sum_{j \geq n+1} \alpha_{2, j} W_{j-1}\left(s_{1}, s_{2}\right)\right) .
$$

We see that Mult $_{2 P} V_{2}^{1}=$ colength $I$ is equal to the intersection number of two algebroid curves at the origin in the $s_{1}, s_{2}$-plane. We will compute this number (Result 6.1).

Considering $s_{1}, s_{2}$ as elementary symmetric functions in two formal replicas $t_{1}, t_{2}$, we have by formula (3.5)

$$
W_{n-1}\left(s_{1}\left(t_{1}, t_{1}\right), s_{2}\left(t_{1}, t_{2}\right)\right)=\prod_{r=1}^{n-1}\left(t_{1}-\varepsilon_{n, r} t_{2}\right)
$$


where $\varepsilon_{n, r}=e^{2 \pi r i / n}$. By standard arithmetic this gives

$$
\begin{gathered}
W_{n-1}\left(s_{1}, s_{2}\right)=\prod_{r=1}^{(n-1) / 2}\left(s_{1}^{2}-k_{n, r} s_{2}\right) \quad \text { when } n \text { is odd } \\
W_{n-1}\left(s_{1}, s_{2}\right)=s_{1} \cdot \prod_{r=1}^{(n-2) / 2}\left(s_{1}^{2}-k_{n, r} s_{2}\right) \quad \text { when } n \text { is even, } \\
\text { where } k_{n, r}=2+\varepsilon_{n, r}+\varepsilon_{n, r}^{-1} .
\end{gathered}
$$

In any case Mult $_{2 P} V_{2}^{1}$ is the sum of the intersection numbers obtained by intersecting the algebroid curve with the equation

$$
\sum_{j \geq n+1} \alpha_{2, j} W_{j-1}\left(s_{1}, s_{2}\right)
$$

with each of the curves corresponding to the factors of $W_{n-1}\left(s_{1}, s_{2}\right)$ (at the origin).

Formula (6.1) implies:

(a) $W_{j-1}\left(s_{1}, s_{1}^{2} / k_{n, r}\right) \equiv 0$ iff $\varepsilon_{n, r}$ is a primitive $m$ th root of unity for an $m$ dividing $j$;

(b) $W_{j-1}\left(0, s_{2}\right) \equiv 0$ iff $j$ is even.

For each $m \geq 2$ we define

$$
\begin{aligned}
B_{m} & =\min \left\{l \mid m \text { does not divide } l, \text { and } \alpha_{2, l} \neq 0\right\}, \\
r_{m} & =\#\{\text { primitive } m \text { th roots of unity }\}
\end{aligned}
$$

or recursively $r_{m}=m-1-\sum r_{m_{i}}$, where the sum is taken over all $m_{i}$ that divide $m$, except 1 and $m$. We then obtain

\section{Result 6.1.}

$$
\text { Mult }_{2 P} V_{2}^{1}=\sum_{i=1}^{s} \frac{r_{m_{i}}\left(B_{m_{i}}-1\right)}{2}
$$

where $m_{1}, \ldots, m_{s}$ are the positive integers (except 1) dividing $n$.

\section{A NOTE ON WEIERSTRASS POINTS}

Let $V$ be a linear system of rank $r+1$ and degree $d$ on a curve $C$. We will use Theorem 4.2 to prove a well-known formula for the weight (multiplicity) of a rank $l+1$ Wronskian point of $V, 0 \leq l \leq r$. A rank $r+1$ Wronskian point is a Weierstrass point.

First we will define our terms, without making any assumptions on the characteristic of $K$. Consider the map

$$
\phi_{l}: C \rightarrow C^{(l+1)}
$$

where $\phi_{l}(P)=(l+1) P$, for $P \in C$. 
Definition 7.1. (a) We say that $V$ is classical if $\phi_{l}^{-1}\left(V_{l+1}^{1}\right)$ is a finite set for $0 \leq l \leq r$.

(b) Assume $V$ is classical. We define the (finite) rk $l+1$ Wronskian scheme of $V$ as $T_{l}=\phi_{l}^{-1}\left(V_{l+1}^{1}\right)$.

(c) We define the (finite) Weierstrass scheme of $V$ as $T_{r}$. The points of $T_{r}$ are denoted by Weierstrass points of $V$.

Let $P$ be an arbitrary point of $C$, and let $t$ be a local parameter of $C$ at $P$. Then there are uniquely determined integers (not depending on the choice of $t) h_{0}<h_{1}<\cdots<h_{r}$ such that there are sections $X_{0}, \ldots, X_{r}$ spanning $V$ with local parametrizations

$$
X_{0}=\sum_{j \geq h_{0}} \alpha_{0, j} t^{j}, \ldots, X_{r}=\sum_{j \geq h_{r}} \alpha_{r, j} t^{j},
$$

with $\alpha_{i, h_{i}} \neq 0$, for $i=0, \ldots, r$. The integers $h_{0}, \ldots, h_{r}$ are called the Hermite invariants of $V$ at $P$. If $V$ is classical, then $h_{i}=i$, for $i=0, \ldots, r$, for all but a finite set of points on $C$.

We now give our result:

Proposition 7.2. Assume char $K=0$ or char $K \geq l+1$, and that $V$ is classical. Then the multiplicity of $T_{l}$ at $P$ is $\sum_{i=1}^{l}\left(h_{i}-i\right)=\sum_{i=0}^{l} h_{i}-l(l+1) / 2$.

Comment. This is essentially [L2, Theorem 15, ii]. In [L2, Theorem 15, i] one proves that if char $K=0$, or $\operatorname{char} K \geq d+1$, then $V$ is classical.

Proof. By Theorem 4.2 we have

$$
\hat{\mathscr{O}}_{V_{l+1}^{\prime},(l+1) P} \approx K\left[\left[s_{1}, \ldots, s_{l+1}\right]\right] / J
$$

where $J$ is generated by the $l+1$-minors of

$$
M=\left[\begin{array}{ccc}
\sum_{j \geq h_{0}} \alpha_{0, j} W_{j}(\underline{s}) & \ldots & \sum_{j \geq h_{r}} \alpha_{r, j} W_{j}(s) \\
\vdots & & \vdots \\
\sum_{j \geq h_{0}} \alpha_{0, j} W_{j-l}(\underline{s}) & \ldots & \sum_{j \geq h_{r}} \alpha_{r, j} W_{j-l}(s)
\end{array}\right]
$$

The map $\phi_{l}: C \rightarrow C^{(l+1)}$ induces a map

$$
\phi_{l}^{*}: \hat{\mathscr{O}}_{C^{(l+1)},(l+1) P} \approx K\left[\left[s_{1}, \ldots, s_{l+1}\right]\right] \rightarrow \hat{\mathscr{O}}_{C, P} \simeq K[[t]]
$$

such that for $k=1, \ldots, l+1$ we have $\phi_{l}^{*}\left(s_{k}\right)=s_{k}(t, \ldots, t)$ where $s_{k}$ is the $k$ th elementary symmetric function in $l+1$ variables. From formula (3.5) we have

$$
\phi_{l}^{*}\left(W_{j}(\underline{s})\right)=W_{j}\left(\phi_{l}^{*} s_{1}, \ldots, \phi_{l}^{*} s_{l+1}\right)=\left(\begin{array}{c}
j+l \\
l
\end{array}\right) \cdot t^{j} \quad \text { for all } j .
$$

This implies that

$$
\hat{\mathscr{O}}_{T_{l}, P} \simeq K[[t]] / \phi_{l}^{*}(J)
$$


where $\phi_{l}^{*}(J)$ is generated by the $l+1$-minors of the matrix

$$
\left[\begin{array}{ccc}
\sum_{j \geq h_{0}} \alpha_{0, j}\left(\begin{array}{c}
j+l \\
l
\end{array}\right) t^{j} & \ldots & \sum_{j \geq h_{r}} \alpha_{r, j}\left(\begin{array}{c}
j+l \\
l
\end{array}\right) t^{j} \\
\vdots & \vdots \\
\sum_{j \geq h_{0}} \alpha_{0, j}\left(\begin{array}{l}
j \\
l
\end{array}\right) t^{j-l} & \cdots & \sum_{j \geq h_{r}} \alpha_{r, j}\left(\begin{array}{l}
j \\
l
\end{array}\right) t^{j-l}
\end{array}\right] .
$$

The multiplicity of $T_{l}$ at $P$ is the lowest number $m$ such that there is a term $t^{m}$ in one of the minors generating $\phi_{l}^{*}(J)$.

The $l+1$-minor consisting of the $l+1$ first columns of (7.1) can be written as $\sum_{j \geq m} c_{j} t^{j}$, where $m=h_{0}+\left(h_{1}-1\right)+\cdots+\left(h_{l}-l\right)=\sum_{i=0}^{l}\left(h_{i}-i\right)$. Clearly no term $t^{n}$, with $n<m$, is contained in any of the generators of $\phi_{l}^{*} J$. Hence we have proved the proposition if we can show that $c_{m}$ is nonzero. We have

$$
c_{m}=\alpha_{0, h_{0}} \cdots \alpha_{l, h_{l}} \cdot\left|\begin{array}{ccc}
\left(\begin{array}{c}
h_{0}+l \\
l
\end{array}\right) & \cdots & \left(\begin{array}{c}
h_{l}+l \\
l
\end{array}\right) \\
\left(\begin{array}{c}
h_{0}-1+l \\
l
\end{array}\right) & & \left(\begin{array}{c}
h_{l}-1+l \\
l \\
\vdots
\end{array}\right. \\
\vdots \\
\left(\begin{array}{c}
h_{0} \\
l
\end{array}\right) & \cdots & \left(\begin{array}{c}
h_{l} \\
l
\end{array}\right)
\end{array}\right| .
$$

The proposition follows from the following lemma:

\section{Lemma 7.3.}

$$
\left|\begin{array}{ccc}
\left(\begin{array}{c}
h_{0}+l \\
l
\end{array}\right) & \ldots & \left(\begin{array}{c}
h_{l}+l \\
l
\end{array}\right) \\
\vdots & & \vdots \\
\left(\begin{array}{c}
h_{0} \\
l
\end{array}\right) & \ldots & \left(\begin{array}{c}
h_{l} \\
l
\end{array}\right)
\end{array}\right|=\left|\begin{array}{ccc}
\left(\begin{array}{c}
h_{0} \\
0
\end{array}\right) & \ldots & \left(\begin{array}{c}
h_{l} \\
0
\end{array}\right) \\
\left(\begin{array}{c}
h_{0} \\
1
\end{array}\right) & \ldots & \left(\begin{array}{c}
h_{l} \\
1
\end{array}\right) \\
\vdots & & \vdots \\
\left(\begin{array}{c}
h_{0} \\
l
\end{array}\right) & \ldots & \left(\begin{array}{c}
h_{l} \\
l
\end{array}\right)
\end{array}\right| .
$$

Comment. In [L1, Lemma 9] one shows that the determinant to the right is $\prod_{0 \leq j<i \leq l}\left(h_{i}-h_{j}\right) \cdot 1 / \prod_{i=1}^{l} i !$, which is nonzero.

Proof of Lemma 7.3. In the first row set

$$
\left(\begin{array}{c}
h_{j}+l \\
l
\end{array}\right)=\left(\begin{array}{c}
h_{j}+l-1 \\
l-1
\end{array}\right)+\left(\begin{array}{c}
h_{j}+l-1 \\
l
\end{array}\right) \text { for } 0 \leq j \leq l
$$

Since the entries in the second row are $\left(\begin{array}{c}h_{j}+l-1 \\ l\end{array}\right)$, these terms can be deleted in the first row. In this way the entries in row $k+1$ can be changed from $\left(\begin{array}{c}h_{j}+l-k \\ l\end{array}\right)$ to $\left(\begin{array}{c}h_{j}+l-k-1 \\ l-1\end{array}\right)$ for $k=0, \ldots, l-1$ and $j=0, \ldots, l$. Then start at the top again and treat all but the two last rows the same way once more. When the top row has been treated this way $l$ times, we end up with the desired determinant. This completes the proof of Lemma 7.3 and also of Proposition 7.2.

Corollary 7.4. Assume char $K=0$, or char $K \geq r+1$. Then the multiplicity of $P$ as a Weierstrass point is $\sum_{i=0}^{r}\left(h_{i}-i\right)$.

Remark. It is a well-known fact that the total length of $T_{r}$, that is, the sum of the multiplicities of the Weierstrass points, is

$$
((g-1) r+d)(r+1), \text { where } g \text { is the genus of } C \text {. }
$$


This follows from [A-C-G-H, pp. 345 and 358] where $k=C$.

Nonclassical linear systems. What happens if we impose no restrictions on char $K$ ? This question has been answered in a very satisfactory way in [L2], and we would be happy to reproduce some of the results in [L2] using our setup. It seems, however, that our methods are too crude when $0<\operatorname{char} K<d$. Still, we will add a few words about this case.

Let $h_{0}, \ldots, h_{r}$ be the Hermite invariants of $V$ at a point $P$ of $C$. On an open set of $C$ the Hermite invariants are constant with values $b_{0}, \ldots, b_{r}$. When $\operatorname{char} K=0$ or char $K>d$, we have $b_{i}=i$ for $i=0, \ldots, r$. When $2 \leq \operatorname{char} K \leq d$, we have $i \leq b_{i}<b_{i+1}$ for $0 \leq i \leq r-1$, and $b_{i}$ might or might not be equal to $i$ for all $i$. In this case we have

$$
\phi_{b_{l}}^{-1}\left(V_{b_{l}+1}^{b_{l}-l+1}\right) \text { is a finite set for } 0 \leq l \leq r,
$$

in analogy with Definition 7.1(a).

$\phi_{b_{l}}^{-1}\left(V_{b_{l}+1}^{b_{l}-l+1}\right)$ is also defined as a finite scheme, which we denote by $T_{l}$, and

$$
R=\hat{\mathscr{O}}_{T_{l}, P} \simeq K[[t]] / \phi_{b_{l}}^{*}(J),
$$

where $\phi_{b_{l}}^{*}(J)$ is generated by the $l+1$-minors of the following $\left(b_{l}+1\right) \times(r+1)$ matrix:

$$
\left[\begin{array}{ccc}
\sum_{j \geq h_{0}} \alpha_{0, j}\left(\begin{array}{c}
j+b_{l} \\
b_{l}
\end{array}\right) t^{j} & \ldots & \sum_{j \geq h_{r}} \alpha_{r, j}\left(\begin{array}{c}
j+b_{l} \\
b_{l}
\end{array}\right) t^{j} \\
\vdots & & \vdots \\
\sum_{j \geq h_{0}} \alpha_{0, j}\left(\begin{array}{c}
j \\
b_{l}
\end{array}\right) t^{j-b_{l}} & \ldots & \sum_{j \geq h_{r}} \alpha_{r, j}\left(\begin{array}{c}
j \\
b_{l}
\end{array}\right) t^{j-b_{l}}
\end{array}\right] .
$$

(As usual $\left(\begin{array}{l}a \\ b\end{array}\right)=0$ if $b>a$.)

The multiplicity of $T_{l}$ at $P$ is the length of the ring $R$. One sees that $P \in T_{l}$ iff $\alpha_{l, j}=0$ for $j \leq b_{l}$, that is, iff $h_{l} \geq b_{l}+1$.

Set-theoretically we have: $P$ is a rank $l+1$ Wronskian point in the sense of [L2] iff $P \in \bigcup_{k=0}^{l} T_{k}$. In [L2, Example 1, p. 64] one shows that is is possible that $P \notin T_{l}$ but $P \in T_{k}$ for some $k<l$. Hence the multiplicity we have described for a point of $T_{l}$ is different from the multiplicity described in [L2] for a rank $l+1$ Wronskian point.

\section{REFERENCES}

[A-C-G-H] E. Arbarello, M. Cornalba, P. A. Griffiths, and J. Harris, Geometry of algebraic curves, Vol. I, Springer-Verlag, New York, 1985.

[F] W. Fulton, Intersection theory, Springer-Verlag, New York, 1984.

[G-P] L. Gruson and C. Peskine, Courbes de l'espace projectif, variétés de sécantes, Enumerative Geometry and Classical Algebraic Geometry, Progress in Math., Vol. 24, Birkhäuser, Boston, Mass., 1982, pp. 1-31.

[L] O. A. Laudal, Formal moduli of algebraic structures, Lecture Notes in Math., Vol. 754, SpringerVerlag, Berlin and New York, 1979.

[L1] D. Laksov, Weierstrass points on curves, Astérisque 87-88 (1981), 221-247. 
[L2] _-Wronskians and Plücker formulas for linear systems on curves, Ann. Sci. Norm. Sup. (4) 17 (1984), 45-66.

[LB] P. Le Barz, Formules multisecantes pour les courbes quelconques, Enumerative Geometry and Classical Algebraic Geometry, Progress in Math., Vol. 24, Birkhäuser, Boston, Mass., 1982, pp. 165-197.

[Ma] A. Mattuck, Secant bundles on symmetric products, Amer. J. Math. 87 (1965), 779-797.

[Ma-Ma] A. Mattuck and A. Mayer, The Riemann-Roch theorem for algebraic curves, Ann. Scuola Norm. Sup. Pisa (3) 17 (1963), 223-237.

[WL] T. Wentzel-Larsen, Deformation theory of trisecant varieties, Preprint No. 1, Univ. of Oslo, 1985.

Department of Mathematics, Skidmore College, Saratoga Springs, New York 128660851

Institute of Mathematical Sciences, University of Troms $\varnothing, 9001$ Troms $\varnothing$, Norway 